\title{
Evaluation von Supervision wohin?
}

\author{
Heidi Möller und Michael Märtens
}

\section{Einleitung: Das Gegenstandsproblem}

Supervision ist ein schillernder Gegenstand, was sich schon beim Aufschlagen des Inhaltsverzeichnisses zum Beispiel des 2. Handbuchs von Pühl (1994) zeigt. Die schillernde Vielfalt hat er mit der Psychotherapie gemeinsam und damit verbunden ist eine vergleichbare Fülle an Problemen. Im Gesundheitsreformgesetz sowie im SGB V werden Anbieter von medizinischen und psychosozialen Dienstleistungen zur Qualitätssicherung verpflichtet, die auch eine vergleichende Prüfung zwischen Anbietern und die direkte Verbesserungen sowie deren Überprüfung ermöglicht. Wenn wir uns der Forderung stellen wollen, empirisch unterstützte Evaluation von Supervision anzustreben, wie sie im SGB V gefordert wird, dann sieht man sich mindestens vier Gegenstandsbereichen gegenüber, die unterschiedliche Forschungsstrategien erfordern: die Einzel-, Gruppen- und Teamsupervision sowie die Organisationsberatung. Wegen der augenblicklichen ,Ausbreitung von Supervision und einer Diversifizierung der Supervisionsformen und -methoden" (Retzer et al. 1997, S. 240), sind weitere Variationen des Gegenstandes Supervision zu erwarten. Diese zwingen eher zu einer weiteren Spezifizierung von Forschungsstrategien für diese unterschiedlichen Anwendungskontexte. Durch die Entwicklung fragestellungsspezifischer Vorgehensweisen sollten oberflächliche Antworten vermieden werden, die vermeintlich für alle Anwendungskontexte guiltig sind.

Im Bereich der Psychotherapieforschung hat sich eine Differenzierung der Evaluationsindikatoren auf Klientenseite ergeben, die auch für Supervision bedeutsam ist. Insgesamt können drei verschiedene Aspekte in der Evaluation von Supervision als einem Spezialfall von Beratung und Psychotherapie unterschieden werden. Strauß (1998) hat diese drei Aspekte für die Psychotherapieevaluation vergleichend gegenübergestellt. Dabei handelt es sich erstens um die Behandlungszufriedenheit, zweitens um die subjektiv wahrgenommene Zufriedenheit mit der Verbesserung der Symptomatik und drittens um die klinische Relevanz (Jacobson \& Truax 1991) der eingetretenen Verbesserung. Oft zeigt es sich, daß die subjektiv erlebten Besserungen zwar statistisch signifikante Verbesserungen darstellen, die Klienten im Sinne einer klinischen Bewertung immer noch im auffälligen Bereich und damit nicht im Bereich der gesunden Normalbevölkerung liegen (Bankoff \& Howard 1986). Verfolgt man die drei Kurven über den Beratungsverlauf, so zeigt sich, daß die Behandlungszufriedenheit relativ schnell ansteigt und ein relativ hohes Niveau behält. Demgegenüber bleibt die subjek- 
tiv eingeschätzte Zufriedenheit mit der Besserung der Symptomatik etwas hinter dieser Kurve zurück. Was die klinische Signifikanz der Veränderung im Bereich Psychotherapie anbelangt, muß festgestellt werden, daß bei einer Vielzahl der Klienten die erzielten Veränderungen nicht ausreichend sind, um die Patienten psychopathologisch als unauffällig oder ausreichend gebessert zu betrachten (s.a. BMFSF\&J 1994a,b).

Howard und Mitarbeiter untersuchen in ihrem Phasenmodell neben der Besserung des allgemeinen Wohlbefindens und der Symptomatik auch die Verbesserungen der psychosozialen Anpassung. Dabei stellt sich heraus, daß diese am meisten Zeit benötigte (Howard et al 1992, zit. in Grawe 1998). Bei Untersuchungen zur Beziehung zwischen der Zufriedenheit mit der Behandlung und dem Ergebnis, wobei dies vom direkten Klienten selbst oder von unabhängigen Beurteilern eingeschätzt werden kann, zeigen sich nur mäßige Zusammenhänge. Aus der Zufriedenheit mit der Behandlung kann nur schlecht auf den Behandlungserfolg geschlossen werden. Es muß also zwischen zufriedenen Gebesserten und unzufriedenen Gebesserten unterschieden werden. Das gleiche Phänomen findet sich auch bei den Gleichgebliebenen und den Verschlechterten (z.B. Jacobson 1997). Zufriedenheit mit einer Behandlung sagt nur bedingt etwas über die Effektivität der Behandlung.

Aus diesen Befunden muß für die Supervisionsevaluation unbedingt der Schluß gezogen werden, daß über die Zufriedenheit hinaus Kriterien angelegt werden müssen, die die Ergebnisqualität erfassen, also die „supervisorische Relevanz“ operationalisieren. Eine Evaluation kann sich nicht mit der Kundenzufriedenheit zufrieden geben. Bisher ist Kundenzufriedenheit gut belegt, aber die Effektivität noch näher zu bestimmen.

\section{Empirische Untersuchungen zur Effizienz von Supervision:}

An ausgewählten Beispielen der Supervisionsforschung sollen die Fortschritte und Probleme der gegenwärtigen Forschung anhand unterschiedlicher Forschungsfelder dargestellt werden.

\section{Supervision als Therapiedidaktik}

Das Gros der empirischen Supervisionsforschung konzentriert sich auf den Gegenstand der Supervision als Pfeiler der Therapiedidaktik. Dieser Forschungsbereich zeichnet den Weg von Psychotherapie-Ausbildungskandidaten nach, deren Entwicklungsschritte hin zu einer reifen Therapeutenidentität unter Berücksichtigung der jede Stufe kennzeichnenden Bedürfnisse, Kompetenzen und Motivationen des Kandidaten sowie der jeweiligen Supervisor-Variablen, die auf jeder Stufe für den Entwicklungsprozeß des Supervisanden eine Rolle spielen.

Stoltenberg (1981) konzeptualisiert in seinem Counselor Complexity Model einen solchen Vorgang über vier Stadien. Er bezieht sich auf das Supervisionsmodell von Hogan (1964), der vier Entwicklungsstufen von Supervisanden beschreibt: ausgehend von fachlicher Unsicherheit, mangelndem Selbstvertrauen, Methoden- und Instruktionsabhängigkeit auf der ersten Stufe entwickeln die Ausbildungskandidaten Autonomie, persönlichen Stil und tiefere Einsichtsfähigkeit, bis schließlich kollegiale Supervi- 
sion und angstfreies Arbeiten mit Konfrontation sowie ein Wissen um die eigenen therapeutischen Möglichkeiten und Grenzen erreicht ist. Stoltenberg berücksichtigt die systemtheoretischen Überlegungen Hunts (1971), der anhand der kognitiven Entwicklung von Schülern zu einer Beschreibung optimaler Lernumwelten gelangt, die jeweils die Entwicklung hin zur nächsten Stufe erleichtern, wobei es wichtig ist, die sich entwickelnde Person in einer sich verändernden Umwelt zu sehen, deren angemessene Gestaltung die Entwicklung zu höheren Entwicklungsstufen vorantreibt.

Das Counselor Complexity Model stellt auf jeder Stufe die Merkmale des Supervisanden einer jeweils optimalen Umwelt, konzeptualisiert durch Verhaltensvariablen des Supervisors, gegenüber. Der Entwicklungsgang des Supervisanden entspricht dabei in etwa dem oben skizzierten Modell von Hogan. Das optimale Supervisor-Verhalten zeigt sich in der ersten Stufe vom Bereitstellen einer eher normativen Struktur, von der ausgehend erste Autonomiebestrebungen gefördert werden können, hin zu einer zunehmenden Übergabe der Strukturierung des Geschehens an den Supervisanden, bis schließlich das Lehrer-Schüler-Verhältnis in ein kollegiales Verhältnis übergeht, in der die optimale Umwelt aus der gegenseitigen Strukturierung erwächst.

Entwicklungsfördernde Kompetenzen des Supervisors beziehen sich nach Blocher (1983) auf den angemessenen Umgang mit Diskrepanzen zwischen den Kompetenzen des Supervisanden und den jeweiligen Anforderungen der Situation, auf den Umgang mit den Auswirkungen der beruflichen Erfahrungen auf das Selbstwertgefühl des Supervisanden, auf adäquates Feedback, Unterstützung und Struktur und die Integration neuer und alter Verhaltensmuster, etwa durch Schaffung von genügend Möglichkeiten zur Reflexion und zur Festigung neuer Lösungswege.

Das Verständnis des Entwicklungsgeschehens in der Supervision als Herstellung eines entwicklungsfördernden Passungsverhältnisses zwischen Person und Umwelt regte eine Reihe von Forschungsarbeiten an, in denen durch Fragebogenuntersuchungen versucht wurde, die Entwicklung des Supervisanden, Verhaltensweisen des Supervisors und wahrgenommene Effekte der Supervision entlang den im Modell verwendeten entwicklungsbedeutsamen Kategorien in Zusammenhang zu bringen. Dabei können die grundlegenden Annahmen des Entwicklungsmodells bestätigt werden. Die Entwicklungsdimensionen Selbstbewußtsein, Abhängigkeit-Autonomie und Erwerb von theoretischen und praktischen Kenntnissen (McNeill, Stoltenberg \& Pierce 1985), wie sie sich auch in anderen Befunden bei ähnlicher Begrifflichkeit, teilweise noch differenzierterer (Reising \& Daniels 1983) finden lassen, scheinen dabei den Entwicklungsweg des Supervisanden besonders deutlich zu kennzeichnen. Die für jede Entwicklungsstufe günstigen Verhaltensweisen des Supervisors entsprechen im wesentlichen den oben genannten (Heppner \& Roehlke 1984; Miars et al. 1983). Leider gibt es bisher keine Studie, die in einem strengen Gruppenvergleichsdesign versucht hat, die Beschleunigung der Entwicklungsprozesse zu objektivieren, indem zum Vergleich der Effekte auch eine Kontrollgruppe untersucht wurde.

Über den tatsächlichen Supervisionsprozeß finden wir in all den Untersuchungen keine Aussagen. Die Konzepte werden jedoch aufgrund ihres hohen Allgemeinheitsgrades für das Verständnis der Gestaltung optimaler „Supervisionsprozesse“ Berücksichtigung finden. Bei allen Forschungsansätzen fällt auf, daß der Begriff des Supervisionsansatzes oft hybrid verwendet wird. In Ermangelung tragfähiger Supervisionsmodelle orientiert sich Supervision noch häufig an Therapiefolien und stellt oft lediglich 
selbst gestaltete Praxis (Zielke 1982) dar, die sich „mit Intuition und gesundem Menschenverstand“ (Holloway et al. 1989) vollzieht. Bernard \& Goodyear (1992) bezeichnen die Übertragung von Therapiemodellen auf die Supervisionsprozesse als ,no-model". Sie sehen die Gefahr, daß Supervisanden wie Klienten behandelt werden, da weder ein konzeptuelles Modell für den Supervisionsprozeß vorliegt, noch die Aufgaben der ,selbsternannten“ Supervisoren in der Therapieszene klar umrissen sind.

Auckenthaler (1995) bemängelt das Fehlen einer qualitativ orientierten empirischen Erforschung der Wirksamkeit von Supervision. Supervision sei als Qualitätssicherung der Psychotherapie weitestgehend anerkannt, ,... obwohl entsprechende Wirksamkeitsnachweise fehlen" (ebd. S. 9). Wir möchten uns Auckenthaler anschließen, wenn sie feststellt, daß die Frage: „Was geschieht wirklich in Supervisionen?“ bislang noch unzureichend beantwortet ist. Auckenthaler untersucht Gruppensupervisionssitzungen von Ausbildungskandidaten und ergänzt ihren qualitativ-empirischen Ansatz durch Gruppendiskussionen mit Supervisoren. Dabei geht sie u.a. der Frage nach, ob (und wie) Supervisanden im Verlauf der Supervision entstandene „Verstehenshypothesen" und deren Einfluß auf den Verlauf der Therapie überprüfen und wie mit Kritik umgegangen wird. Die subjektive Perspektivität der Beteiligten wird einbezogen, indem die Autorin untersucht, welche Bedeutung die Handelnden selbst ihren Aktivitäten zuschreiben, wie sie ihr Handeln begründen und wie sie selbst ihre Supervision bewerten.

Ihr theoretischer Hintergrund stellt ausschließlich psychologische Literatur zur Supervision von Psychotherapie vorrangig aus den USA dar. So ist der Tenor ihrer Ergebnisse die Ermunterung von Supervisoren aus der Richtung der klientenzentrierten Psychotherapie, ihre Rolle als Lehrer ernster nehmen zu dürfen. Gute Klientenzentrierte Supervisoren, so stellte sie heraus, fordern und konfrontieren die Ausbildungskandidaten, sie geben mehr belehrende als unterstützende Interventionen, als sie es in therapeutischen Situationen täten. Sie erklären, setzen Grenzen und bewerten Aussagen von Supervisanden auch als falsch. Man kann sich Auckenthaler sicher nur anschließen, wenn sie ausführt, daß die Ausbildung zum Psychotherapeuten keine gleichzeitige Qualifikation zum Supervisor bedeutet. Es geht um ergänzende Wissensbestände, wie zum Beispiel die institutionelle Einbindung von Supervisanden - eine Erkenntnis, die richtig jedoch nicht sonderlich neu ist -, die besondere Berücksichtigung erfordert.

Der Mangel an spezifischem Wissen ist insofern beklagenswert, da Supervision immer mehr zum zentralen Bestandteil beraterischer und psychotherapeutischer Arbeit wird. Im Rahmen der „Internationalen Studie zur Entwicklung von Psychotherapeuten und Psychotherapeutinnen“ (Ambühl 1994; Ambühl u.a. 1995; Orlinsky u.a. im Druck) berichten 74\% von 581 befragten PsychotherapeutInnen im deutschen Sprachraum, daß sie sich derzeit in Supervision befinden (im Mittel mit fünf ihrer Fälle). Mit 49\% ist die Gruppensupervision das häufigste Setting (in Einzelsupervision befanden sich 34\%, in kollegialer Supervision 17\% der Befragten). 57\% der TherapeutInnen sind selbst als SupervisorIn tätig, und zwar zumeist für 4-7 andere KollegInnen (zit. nach Willutzki 1995). Die Untersuchung von Mary Burton und Kollegen zeigt, daß wir in England eine vergleichbare Situation vorfinden (Burton, Henderson \& Curtis Jenkins 1998).

Die meistens vorliegenden Untersuchungen fokussieren folgende Aspekte, von denen als typische Auswahl folgende genannt werden sollen: 
- Interaktion zwischen Supervisor und Supervisanden: der Einfluß der Selbstdarstellung des Supervisanden (Ward, Friedlander, Schoen \& Klein 1985),

- die Übereinstimmung in der theoretischen Orientierung von Supervisor und Supervisanden (Putney, Worthington \& McCullough 1992; Steinhelber, Patterson, Cliffe \& LeGoulon 1984),

- Rolle der Geschlechtszugehörigkeit des Supervisors (Nelson \& Hooloway 1990; Petty \& Odewahn 1984; Brodsky 1980; Putney et al. 1992, Erger \& Molling 1991)

- Erfahrungshintergrund des Supervisors (Worthington \& Stern 1985; Marikis, Russel \& Dell 1985)

- Persönlichkeitsmerkmale des Supervisors: expertness, attractiveness, trustworthiness (Goodyear, Abadie \& Efros 1984; Heppner \& Handley 1982; Heppner \& Roehlke 1984)

- Charakteristika des Supervisionsstils: interpersonal sensitivity, task orientation, attractiveness (Friedlander \& Ward 1984).

Die Spezifika von Teamsupervision finden sich in oben genannten Untersuchungen wenig wieder. Die meisten Daten sind in Ausbildungssupervisionen gewonnen worden, wo der Zugriff auf die Daten sicher leichter fällt, die Erkenntnisse für den inzwischen größten Anwendungsbereich, nämlich der Teamsupervision, aber sicherlich nur bedingt uibertragbar sind.

\section{Supervisionsausbildung als Forschungsfeld}

Nach der Beschäftigung mit den Ergebnissen aus dem Bereich Therapieausbildung wenden wir uns jetzt der Vermittlung der Fertigkeiten zu, die in der Supervisionsausbildung selbst vermittelt werden. Dabei handelt es sich also um metakonzeptionelle Lernvorgänge. Van Kessel kritisiert in diesem Zusammenhang, daß Supervion in Deutschland zuwenig unter dem Aspekt des Lernens betrachtet wird, der zugunsten der Erkenntnis vernachlässigt wird. Er betrachtet Lernen als zentralen Vorgang in Supervisionsprozessen (van Kessel 1998).

Von Schreyögg wurde 1994 eine empirische Evaluation von Supervisorenausbildungen eines Integrativen Supervisionsansatzes $(\mathrm{N}=145)$ vorgelegt. Die Kandidaten wurden mit einer Kombination von Polaritätsskalen und Fallbeispielen auf ihren Lernzuwachs nach Abschluß ihres Ausbildungsweges untersucht, der durch Experten-Evaluationen ergänzt wurde. Die Handlungssicherheit bei den Ausbildungskandidaten nahm zu. Sie erweiterten ihre Kompetenzen und Performanzen, nachdem sie ein Durchgangsstadium der Verunsicherung ,durchlitten“ hatten. Nach Abschluß der Ausbildung zeigten sich differenziertere Kategorisierungen und weniger Stereotypisierungen. Ihre Deutungshaltung veränderten sich in Richtung auf mehr Systematik im Vorgehen. Schreyöggs Ergebnisse sprechen für eine Mischung von Berufsgruppen in den Ausbildungsgruppen. Reine Psychotherapeutengruppen verfügten über das geringste Entwicklungspotential, was auch auf die Effektivität multiprofessioneller Teams hinweist.

Die Ausbildung von Supervisoren in Österreich untersuchten Petzold \& Schigl (1996). Eine Supervisionsausbildungsgruppe wurde über vier Jahre hinweg von einer Forscherin begleitet, die den Prozeß auch aus der Perspektive einer teilnehmenden Beobachterin verfolgte. Neben quantifizierenden Erhebungen wurden qualitative Verfah- 
ren (Interviews, Berichte, etc.) eingesetzt. Um auch die Effizienz auf der Klientenebene zu überprüfen, wurden Videoaufzeichnungen vor der Ausbildung mit Aufzeichnungen nach der Ausbildung durch Ratingverfahren auf der Ebene der Performanz verglichen. Durch einen Vergleich mit einer Kontrollgruppe ohne Ausbildung konnte hier die Bedeutung der Ausbildung zumindest operationalisiert durch Indikatoren für eine Verbesserung der Performanz demonstriert werden, ohne allerdings die tatsächlichen Effekte auf die Klienten mitberücksichtigen zu können.

\section{Balintgruppenforschung}

Die Forschungsergebnisse zu Balintgruppen stellen sicherlich den größten Anteil aller Forschungsbefunde dar. Seit ihrer Einführung waren sie wie viele andere psychoanalytische Vorgehensweisen auch Forschungsinstrument und Behandlungsform gleichzeitig, was sich deutlich schon im Werk Balints zeigt (Balint 1957). Da hierzu eine umfangreiche Literatur vorliegt, soll an dieser Stelle auf eine Zusammenfassung verzichtet werden (z.B. Möller \& Märtens 1998).

\section{Empirische Untersuchungen zur Effizienz in unterschiedlichen Kontexten}

Elbing \& Huber (1988) untersuchten Supervision als Programmatik und als subjektiv erfahrene Alltagsrealität. Im Rahmen einer qualitativen Pilotstudie wurden Erziehungsberater nach ihren subjektiven Erfahrungen mit Supervisionsprozessen befragt. Die Notwendigkeit von Supervision wurde stets verbal bekundet, jedoch sahen sich die Mitarbeiter zahlreichen Einschränkungen gegenüber. Neben den persönliche Ängsten und Unsicherheiten wurden Teamschwierigkeiten und institutionelle, hierarchische und organisatorische Aspekte als den Supervisionsprozeß behindernd beschrieben. Die Studie zeigt anschaulich den Status quo supervisorischen Arbeitens, arbeitet allerdings keine Bewältigungsmöglichkeiten heraus.

Filsinger \& Schäfer (1992) untersuchen die Kontextbezogenheit und -determiniertheit von Supervision im Zusammenhang mit der erworbenen Immunschwäche (AIDS). Berichte von Supervisoren wurden daraufhin untersucht, ob und wie und welche Kontextvariablen in die Supervision miteinbezogen wurden. Die Analyse der Defizite in Struktur, Methode und Perspektivität der supervisorischen Arbeit zeigt die Notwendigkeit, neben professionellen und individuell-biographischen Bedingungen institutionelle, versorgungsstrukturelle und gesellschaftliche Faktoren in der Arbeit zu berücksichtigen. Die Autoren betonen sowohl die Wichtigkeit der Feldkompetenz in diesem Bereich, als auch die Notwendigkeit der Organisationsentwicklung und -beratung, damit die Handlungsmöglichkeiten der Berater auch institutionell greifen können. Supervisoren empfanden die Arbeit hier vor allem im Rahmen von Bundesmodellprogrammen als spezifische Herausforderung.

Supervision bzw. Praxisberatung für Lehrergruppen wurde von Cloett (1985) untersucht. Die befragten Berater, Absolventen und Teilnehmer bestätigten die Effizienz berufsbegleitender Supervision für sie Fähigkeit von Lehrkräften, im Beruf zwischenmenschliches Geschehen und eigenes Verhalten unter dem affektiven Aspekt genauer 
wahrzunehmen, in geeigneter Weise zur Sprache zu bringen und in Problemsituationen geeignete Lösungen zu finden. Der kontinuierlichen Evaluation von Praxisberatung durch außenstehende Forscher standen sie eher skeptisch gegenüber.

Neu eingestellte Lehrer untersuchte de Lorent (1992). Der „Praxisschock“ wurde vor allem auf die Disziplinprobleme, ein überaltertes Kollegium sowie die Einarbeitung in neue Fächer zurückgeführt. Junge Lehrer wünschen sich der Untersuchung zufolge vor allem die Einrichtung von Supervisionsgruppen, Training von Durchsetzungskompetenzen und Hilfe bei o.g. Problemen. Eine stärkere Praxisorientierung des Referendariats, intensivere Beschäftigung mit Verhaltensproblemen der Schüler und eine Erhöhung ihrer pädagogischen Kompetenz wurde gefordert. Meidinger (1991) berichtet über eine Evaluationsstudie über zwei Supervisionsgruppen von Lehrerkollegien. Mit Hilfe von Antons Evaluationsbogen sollten Unterschiede zwischen einer Gruppe, die sich ihren Supervisor selbst gesucht hatte, zu einer anderen Gruppe, die den Supervisor zugeteilt bekam, aufgezeigt werden. Das Zustandekommen der Supervisionsgruppe besaß weder prognostischen Wert bzgl. des Beurteilungsverhaltens, noch ließ es Rückschlüsse auf die Motivation der Teilnehmer zu. Die Untersuchung ist in Hinblick auf die Mystifizierung der freien Wahl des Supervisors, wie sie insbesondere in der psychosozialen Szene herrscht, von Interesse, da dieser Aspekt möglicherweise überschätzt wird.

Mutzeck (1988) untersuchte die subjektive Theoriebildung von Lehrern über den mangelhaften Transfer der Inhalte von Fortbildungsveranstaltungen in den Schulalltag. Aus den subjektiven Annahmen, die inhaltsanalytisch ausgewertet wurden, entwickelte Mutzeck ein alltagsnahes Handlungsmodell zur effektiven Gestaltung von Fortbildungs- und Supervisionskursen.

Denner (1998) kombiniert einen Fragebogen zur jeweiligen Supervisionssitzung mit einem halbstrukturierten Interview, um die Konzeption eines Beratungsangebots für Lehrer zu evaluieren. Sowohl den Beratern als auch den Teilnehmern der Beratungsgruppen wird nach der Sitzung ein Fragebogen vorgelegt. Die Ergebnisse zur Frage der Effizienz von Beratungsgruppen an Schulen: Entstehung - Prozeß - Wirkung" stehen in Gänze noch aus. Denner gelingt es jedoch, durch ihren methodenpluralen Zugang aus der reinen Legitimationsforschung herauszufinden. Durch die Interviews sind fundierte Daten zu erwarten, um Widerstandsphänomene in der Institution Schule besser verstehen und in der Passung des Angebots berücksichtigen zu können. Ihr methodischer Absatz scheint uns sinnvoll einsetzbar zur Selbstevaluation von Supervisionsgruppen.

Kinzl (1990) evaluiert eine fünfjährige Balintarbeit mit einer Gruppe von Logopädinnen. Als spezifische Wirkung läßt sich die bewußtere Gestaltung der Beziehung zum Patienten nachweisen. Als unspezifische Wirkfaktoren werden die Erweiterung der beruflichen Identität, das Integriertsein und Verstandenwerden in der Gruppe sowie das Erkennen psychosozialer und psychosomatischer Zusammenhänge genannt.

\section{Geschlechtsrollenfragen}

In neuster Zeit sind einige Arbeiten zur Geschlechtsrollenfrage in der Supervision veröffentlicht worden (Erger \& Molling 1991). Das Merkmal der Geschlechtzugehörigkeit ist sowohl für die Rolle des Supervisanden als auch für die Rolle des Supervisors ver- 
nachlässigt worden. Conen (1993) zeigte in einer Befragung von insgesamt 33 Supervisanden:

- daß Männer mehr als Frauen auf ein positives Gruppenklima angewiesen sind,

- daß sowohl für Frauen als auch für Männer die Leitung einer Supervisionsgruppe durch eine Frau nicht unproblematisch erscheint,

- daß Frauen bei der Supervisorin vor allem Kompetenz und ein Modell suchen, bei einem Supervisor die Anerkennung in der Rolle der erwerbstätigen Frau im Vordergrund steht und

- daß Männer bei einem Supervisor viel Wert auf Kompetenz legen und bei weiblichen Supervisorinnen eher Bestätigung und Anerkennung suchen.

Hege (1990) wandte sich der Frage der Beziehungsgestaltung von Frauen und Männern mit ihrem jeweiligen Klientensystem zu und suchte nach typischen Gestaltungsmerkmalen der supervisorischen Situation unter weiblicher bzw. männlicher Leitung. An einem Fallbeispiel weist sie die stärkere Abgrenzungsfähigkeit von Männern und die Einnahme der Mutterrolle durch Frauen nach, von der sich männliche Teilnehmer von Supervisionsgruppen wiederum bedroht sehen. Frauen scheinen ihr zufolge eine symbiotischere Beziehungsführung zu verteidigen.

Insgesamt erscheint es augenblicklich fraglich, ob Untersuchungen zu „Genderfragen" im Bereich der Supervision sinnvoll und notwendig sind, da die Ergebnisse kaum spezifische Erkenntnisse für den Kontext Supervion erwarten lassen, sondern vor allem allgemeine Wahrnehmungs- und Reaktionsstrukturen offenlegen, die von allgemeinerer Bedeutung sind.

\section{Akzeptanz von Supervision}

Berufspolitisch von besonderem Interesse ist die Beantwortung der Frage nach der schon erreichten Verbreitung von Supervision in unterschiedlichen Anwendungsfeldern und den Möglichkeiten des gezielten Einsatzes in anderen Bereichen.

Berker (1989) befragte 46 Institutionen, zur Relevanz der Supervision. Supervision wurde allgemein für notwendig und förderungswürdig befunden. Bei der Frage der Bedingungen einer erfolgsversprechenden Supervision wurden die Thesen der Bereitstellung eines Ortes und Interventionsinstrumentariums zur Korrektur, Entwicklung und ständiger Innovation beruflichen Tuns und dessen institutionellen Bedingungen aufgestellt. Auf diese Weise kann Supervision zur Entstehung einer neuen Arbeits- und Gesellschaftskultur beitragen.

Kühl \& Pastäniger-Behnken (1998) legen eine Erkundungsstudie vor, die untersucht, inwieweit Supervision in den neuen Bundesländern bereits implementiert werden konnte. Sie zeigen, welche Bedingungen fördernden oder hindernden Charakter haben und wie sich die Supervisionsverbreitung und deren Funktion in den einzelnen Feldern darstellt. Ihr Ziel ist es, das Beratungsangebot in Feldern sozialer Arbeit zu verbessern und Supervision als Beitrag zur Qualitätssicherung zu implementieren. An dieser Stelle scheint es wenig sinnvoll, die Ergebnisse im Einzelnen zu referieren. Viel interessanter erscheint es, ihr gegenstandsangemessenes methodisches Vorgehen darzustellen. Sie haben das Supervisions-Evaluations-Inventar (SEI) von Schneider \& Müller (1995) sowie dessen Weiterentwicklung durch Beer (1997) um eine Interview- 
studie zu entscheidenden Episoden des Supervisionsprozesses ergänzt, um die praxisrelevanten Anstöße zu erfassen. Insbesondere die Kombination von quantitativen und qualitativen Verfahren (repräsentative Fragebogenuntersuchung in Kombination mit Narrationen beruflich bedeutsamer Schlüsselerlebnisse) zeigt, daß in der Supervisionsforschung das Chisma Hermeneutik (Erzählen) versus Quantifikation (Zählen) überwunden werden kann.

\section{Wirksamkeit auf der Klientenebene}

An vielen Stellen wird der Mangel an Nachweisen der Effizienz für Klienten beklagt, der tatsächlich beunruhigend ist, und mehr Forschung für den „Endabnehmer" (Buer 1998, S. 13) gefordert. Für den Forschungsnotstand können mehrere Gründe verantwortlich gemacht werden, die unter anderem durch die umfangreichen Bemühungen der Psychotherapieforschung deutlich werden:

Es ist schwierig, die Effekte unterschiedlicher psychotherapeutischer Methoden zu ermitteln (z.B.Wampold 1997, Roth \& Fonagy 1996). Noch schwerer muß es sein, die Effekte einer Modulation oder Verfeinerung dieser Methoden, wie sie in der Supervision von Psychotherapeuten und Beratern erfolgt, zu erfassen. Es handelt sich hier oft um eine mikroskopische Veränderung im Gesamtprozeß, wenn dieser Prozeß auf der Klientenebene untersucht wird.

Die Therapieforschung und die Ergebnisse der Supervisionsforschung zu den Auswirkungen und Bedingungen erfolgreicher Supervision aus der Perspektive der Supervidierten in der Einzel- und Gruppensupervision verweisen auf die zentrale Bedeutung vorwiegend technikunabhängiger Fähigkeiten der Beziehungsgestaltung und von Persönlichkeitseigenschaften. Crits-Christoph \& Mintz (1991) zeigen, daß es prinzipiell erfolgreiche und weniger erfolgreiche Therapeuten gibt, wobei es sich hier nicht um ein unveränderbares Merkmal handeln muß.

In einer Reanalyse wurden die Ergebnisse häufig zitierter Outcome-Vergleichsstudien mit den Originaldaten daraufhin analysiert, welchen Beitrag (erklärte Varianz) die Therapeutenvariable zum Gesamtergebnis in positiver und negativer Hinsicht beiträgt (Crits-Christoph et al. 1991). Dabei stellt sich heraus, daß einige Therapeuten einen besonders großen Anteil an Besserungen bei Klienten bewirken konnten, also die Überlegenheit des therapeutischen Verfahrens vor allem auf diese Therapeuten zurückzuführen ist, die ihre Therapien erfolgreich abschlossen.

Da solche Beziehungsgestaltungsfähigkeiten nur langfristig verändert werden, können sich kurzfristige Supervisionseffekte kaum in einer therapeutischen Erfolgsstatistik auf Klientenebene niederschlagen. Ein Nachweis solcher Effekte in Mittelwertunterschieden zwischen Klientengruppen ist kaum zu erwarten.

Ganz unterschiedliche therapeutische Vorgehensweisen führen zu guten Ergebnissen bei manchen Klienten und scheitern bei anderen. Trotz einiger klinischer Hinweise auf spezifische Indikationen bei festumschriebenen Störungen (Roth und Fonagy 1996, Lambert \& Bergin 1994), weisen alle Methoden Grenzen auf. Auch Behandlungen mit Supervision können nicht die Grenzen einer Behandlungsmethode transzendieren. Augenblicklich muß man davon ausgehen, daß noch unentschieden ist, ob durch das Einschwören auf eine bestimmte methodische Vorgehensweise entsprechend einer therapeutischen Schule die Effektivität gesteigert werden kann oder derartige Verbesserun- 
gen gerade durch eine Erweiterung und Flexibilisierung der Vorgehensweise zu erwarten ist.

Liegt der Haupteffekt von Fallsupervision im Nutzen für den konkreten Fall, der supervidiert wurde, oder aber im Gewinn an Fähigkeiten, die zur allgemeinen Kompetenzverbesserung beitragen? Wenn klinische Studien unter Praxisbedingungen durchgeführt werden, dann wird unter normalen Supervisionsbedingungen nur ein verschwindend geringer Anteil aller Fälle überhaupt in die Supervision eingebracht. Unter diesen Bedingungen kann nicht davon ausgegangen werden, daß diese wenigen überhaupt in die Supervision eingebrachten Fälle statistisch in der Gesamtstichprobe nachher einen wesentlichen Unterschied ausmachen. Würden die supervidierten Fälle mit den nicht supervidierten Fällen verglichen, müßten diese nach dem Zufallsprinzip ausgewählt werden, was der klinischen Realität zuwiderläuft.

Durch Supervision kann einerseits ein Effekt auf den Einzelfall, der supervidiert wurde, vorliegen, oder die Bedeutung liegt im Transfergewinn für andere Fälle. Dies verweist auf eine unlösbare methodische Schwierigkeit. Beide Aspekte können nicht getrennt und kontrolliert werden. Ob, und in welchem Umfang sie vorliegen, muß augenblicklich angesichts der geringen Effekte als ungeklärt gelten, obwohl von beiden Effekten ausgegangen wird. Dies läßt sich an Einzelfällen gut demonstrieren, so daß man von diesen Effekten ausgeht, ohne sie im Vergleichsgruppendesign notwendigerweise zu finden. Einen besonderen Fähigkeitsgewinn durch Supervision in allgemeinen Psychotherapieergebnissen nachweisen zu können, erscheint augenblicklich unrealistisch.

Stein und Lambert gelang es 1995 nur schwer metaanalytisch in 36 Studien überhaupt nachzuweisen, daß umfangreiche und teure Therapieausbildungen zumindest für bestimmte Klientengruppen im Outcome-Vergleich mit weniger ausgebildeten Therapeuten einen Kompetenzgewinn aufweisen. Der gefundene Effekt war statistisch zwar nachweisbar, aber in seiner Gößenordnung wenig aussagekräftig. Weil dieser Effekt auch mit dem Umfang der beruflichen Erfahrung kovarierte, kann hieraus der Gewinn durch eine Ausbildung auf dieser Ebene nur schwer als nachgewiesen gelten. Die Daten schließen ihn aber auch nicht aus.

Bei der Suche nach Effekten bei Klienten hat es der Forscher mit einer „homöopathischen Spurensicherung “ (Möller \& Märtens 1998) zu tun. Die Effekte werden einmal durch die Aufnahmekapazität und Aufnahmebereitschaft des Superviderten, dann wieder durch dessen Performanz und anschließend durch die vom Supervisor nicht zu beeinflussende Aufnahmebereitschaft eines Klienten oder eines Klientensystems bestimmt. Es handelt sich um indirekte Effekte, bei denen durch die Transformationsprozesse keine Konzentration der Effekte erfolgt. Somit ist verständlich, daß bei der Suche nach den Effekten auf dieser Ebene erhebliche Anstrengungen der Studiendesigns nötig sind, um nach spezifischen positiven und negativen Wirkungen zu suchen, die mit den üblichen Erfolgskriterien nicht erfaßt werden, sofern sie überhaupt vorhanden sind.

Demgegenüber konnte die Frage, wieviel besser geht es den Supervidierten und welche Supervisionsmerkmale sind Voraussetzungen für diese Effekte, viel besser beantwortet werden. Hier handelt es sich um Effekte auf die direkten Kunden, während die Klienten die indirekten Kunden sind. Vielleicht sind die wesentlichsten Effekte der Supervision vor allem bei diesen direkten Kunden zu suchen und genügen als Legitimation schon, da sie wesentliche Funktionen berufliche Praxis fördern und präventive 
Funktionen für die Supervidierten erfüllen. Sollte sich herausstellen, daß Supervision eine sehr erfolgreiche Burnout-Prophylaxe ist (Fengler1994), so wäre dies ein völlig ausreichender Befund um Supervion in vielen Arbeitsfeldern zu legitimieren. Ihre Rechtfertigung ergibt sich aus den Kostenersparnissen für den Arbeitgeber in Form gesparter Fehlzeiten, geringerer Mitarbeiterfluktuation und dem Gewinn an Gesundheit und Lebensfreude bei den Mitarbeitern.

\section{Untersuchung bedeutsamer Phänomene im Supervisionsprozeß}

1. Supervisionsforschung könnte Episoden des Supervisionsprozesses, die von allen Beteiligten als veränderungsrelevant eingeschätzt wurden, ins Zentrum der Untersuchungen stellen. Die geforderte und notwendige Subjektsicht käme zum Tragen, ohne daß die dahinter liegenden Latenzen vernachlässigt werden müßten. Die Verschränkung der bewußten und unbewußten Anteile von Supervisor und Supervisanden könnten in den Blick gerückt und abgebildet werden. Auf diese Weise fänden wir einen sinnverstehenden, hermeneutischen Zugang zu entscheidenden Supervisionssequenzen, gerade ein solcher scheint uns für die Erforschung supervisorischen Tuns angemessen zu sein.

2. Auch Teams weisen pathogene Überzeugungen auf (vgl. Weiss 1993). Pathogene Überzeugungen werden zumeist unbewußte Überzeugungen genannt, die sich in ihrer Wirkung als pathogen herausstellen. Zum Beispiel könnten Teammitglieder die Realisierung eigener Wünsche als Bedrohung der Klinikstruktur bewerten. Auch negative Arbeitsbeziehungserfahrung an aktuellen oder vormaligen Arbeitsplätzen können solche kognitiven Aktivitäten erzeugen, ,deren Endprodukte pathogene Überzeugungen sind“ (Volkart 1995, S. 129). Negative Affekte, Hilflosigkeitsgefühle (das Team fühlt sich als Ganzes nicht kompetent) aktivieren Abwehrprozesse und unterstützen eine resignative Haltung dem eigenen Tun gegenüber. Weitere Bespiele pathogener Überzeugungen sind „Überlebensschuldgefühle“ in Zeiten ansteigender Arbeitslosigkeit und eventueller Entlassung von Kollegen aufgrund schwindender Finanzen. Schuldgefühle, Scham und Angst engen den Handlungsspielraum eines Teams ein und lassen keine Arbeitzufriedenheit aufkommen. Andere Mitarbeiter können sich eventuell aus falsch verstandener Loyalität und Identifikation nicht aus und von der Institution lösen.

3. All das sind Beispiele pathogener Überzeugungen, deren Wirkweise in der Supervision bearbeitet werden können und die zu erforschen interessant ist. Welche Bewältigungsphantasien prägen das Team? Wie realisiert sich der Plan des Teams, den Supervisor von den eigenen pathogenen Ansichten zu überzeugen und gleichzeitig von ihnen ,erlöst" werden zu wollen? Vollziehen Supervisanden einen unbewußten „Rollenumkehrtest“ und bringen den Supervisor in die Rolle, in die sich das Team durch ihr Klientel gedrängt sieht? Welche Interventionen des Supervisors sind hilfreich? Sind es die ,pro-plan-orientierten“ oder die ,,anti-plan orientierte" Reaktionen des Supervisors, die progressive Verhaltensänderung bewirken?

4. Die subjektiven Theorien und Attributionsmuster der Supervisanden generell erscheinen uns ebenfalls untersuchungswert. Welche Attributionsmuster finden wir und wie wirken sie sich in der Supervisionssitzung aus? Werden Arbeitsstörungen 
der eigenen Person, der Klinik als solcher oder dem Behandlungssetting zugeschrieben? Wie lassen sich subjektive Theorien verändern bzw. erweitern? Wie verschränken sie sich mit den leitenden Heurismen der Supervisoren?

5. „Der Anfang setzt die Struktur“, ausgehend von diesem alten Gestaltgesetz könnten Forscher sich die Frage stellen, was die ersten fünf Minuten von Supervisionsitzungen bereits beinhalten. Was sagt die Verhandlung darüber, was Gegenstand der Sitzung werden kann, bereits über den folgenden Fall und die Team- und Institutionsdynamik aus? Eine derartige Forschung könnte für den Supervisor nutzbare Überlegungen in Gang setzen.

6. Auch kritische Phasen in der Supervision könnten ins Zentrum supervisorischer Forschung gerückt werden. Vermutlich beinhalten gerade kritische Phasen des Supervisionsprozesses die relevanten unbewußten Bedeutungen. Wie werden in kritischen Prozeßabschnitten neue Sinninterpretation geschaffen? Ist der Supervisionswunsch selbst ein Abbild einer kritischen Phase im Therapieverlauf oder in der Teamdynamik?

Heppner \& Roehlke (1984) untersuchten Episoden des Supervisionsprozesses, die von allen Beteiligten als besonders bedeutsam, d.h. veränderungsrelevant, eingeschätzt wurden. Die Supervisanden wurden am Ende eines Ausbildungsabschnittes nach solchen kritischen Ereignissen und ihrer subjektiven Bedeutsamkeit befragt. Die häufigsten kritischen Ereignisse bezogen sich auf emotionale Bewußtheit/Konfrontation, Unterstützung und Zweck/Richtung in der therapeutischen Arbeit. Supervisanden mit wenig und mittlerer Erfahrung unterscheiden sich von Supervisanden mit hohem Erfahrungsstand insofern, als die beiden ersten mehr kritische Ereignisse bezüglich Selbstbewußtheit und Unterstützung, die letzten mehr bezüglich des Einflusses von Persönlichkeitsaspekten auf die therapeutische Arbeit berichten. Somit würde der Entwicklungsfortschritt des Supervisanden eine Bewegung von Unterstützung- und Selbstbewußtheitsthemen hin zur Thematisierung von persönlichen Prozessen und aufdeckenden Interaktionen beinhalten.

Die geforderte und notwendige Subjektsicht käme zum Tragen, ohne daß die dahinter liegenden Latenzen vernachlässigt werden müßten. Die Verschränkungen der bewußten und unbewußten Anteile von Supervisor und Supervisanden könnten in den Blick gerückt und abgebildet werden. Auf diese Weise fänden wir einen sinnverstehenden, hermeneutischen Zugang zu entscheidenden Supervisionssequenzen, gerade ein solcher scheint für die Erforschung supervisorischen Tuns angemessen zu sein.

\section{Nebenwirkungen und Risiken von Supervision als Erkenntnisquellen}

„Risiko heißt: Eine Entscheidung kann noch fallen!“ schreibt Matthias Greffrath und führt weiter aus, das der Homo sapiens das Tier ist, das Risiken eingehen kann. „, Die riskante Jagd ernährt die Sippe, der Verzehr der Vorräte vor einem unerwartet langen Winter ist tödlich, das Überqueren des verschneiten Gebirges die Alternative zum schnellen Verhungern. Menschen gehen Risiken ein um des Überlebens und des Genusses willen“ (1998, S. 10). Gleiches gilt auch für Supervision. Supervision kann nicht nur die Überlebensfähigkeit und den Genuß psychosozialer Helfer steigern und gleichzeitig die Erfolge auf Seiten der Klienten potenzieren. Beim Versuch, die wie 
auch immer geartete Praxis als Sozialarbeiter, Psychologe, Mediziner oder Verwaltungsleiter zu optimieren, gibt es Risiken. Selbst der, der nichts tut, geht dabei ein Risiko ein. Jede Chance ist ein Risiko, denn sonst wäre sie keine Chance.

In der letzten Zeit wurden - nicht zuletzt wegen eines Legitimationsdrucks - vor allem die Chancen beleuchtet, wenn es um psychosoziale Interventionen geht, zu denen Psychotherapie, Prävention und auch Supervision gehören. Wenn im Zusammenhang mit Supervision eine Unterscheidung zwischen Risiken und Nebenwirkungen getroffen wird, so wird damit ein komplexer Sachverhalt entsprochen. $\mathrm{Da}$ man sich mit diesem Phänomen überhaupt beschäftigen muß, legen leider die empirischen Befunde nahe.

Burten, Henderson und Curtis Jenkins (1998) fanden in ihrer Untersuchung zu den Erfahrungen mit Supervision aus der Sicht der Supervisanden, daß $11 \%$ der Befragten angaben, eine Erfahrung mit ihrem Supervisor gemacht zu haben, die als Mißbrauch der Beziehung mit negativen Auswirkungen beschrieben wird (1998, S. 127). Diese negativen Erlebnisse werden allerdings auf der anderen Seite aufgewogen durch ansonsten ebenso positive Gesamteinschätzungen, wie sie sich auch schon in der Erhebung von Beer und Gediga (1998) finden. Aber auch Beer und Gediga berichten von unerwünschten Effekten. Bei einem der drei Teams, von denen der Dienstvorgesetzte befragt wurde, beklagte dieser, da $\mathrm{B}$ im ,institutionellen Bereich Abläufe erschwert wurden" und sich das Verhältnis zur Leitung nicht verbessert hatte. Aus der Tatsache, daß er dieses Item mit der größtmöglichen Verneinung beantwortet, kann vermutet werden, daß hier möglicherweise eine Verschlechterung eingetreten ist.

Die Schwierigkeiten hierzu, eine klare Aussage zu machen, verweisen auf einen häufig zu beklagenden Mißstand der Studienkonzeption. Sehr oft werden - wie auch in der Untersuchung von Beer und Gediga - differenzierte Vorgaben zur Erfassung einer weiten Pallette positiver Wirkungen in vier Bereichen (eigene Person, Klienten, Kollegenkreis, Institution) gemacht. Negative Phänomene werden wesentlich weniger oder überhaupt nicht explizit formuliert. Durch eine Verweigerung der Zustimmung zu einer positiven Formulierung, kann aber noch nicht der Umkehrschluß getroffen werden, daß diese Verweigerung auf einen negativen Sachverhalt hindeutet. Werden negative Phänomene nicht explizit erfragt, kann über deren Vorhandensein oder Abwesenheit nichts gesagt werden. In Anlehnung an Wittgenstein, der einmal formulierte, worïber man nicht reden kann, darüber kann man nur schweigen, müßte man dann sagen, daß man zu negativen Effekten, zu denen man keine Fragen gestellt hat, leider auch nichts sagen kann.

Beer und Gediga finden die Befürchtung Nellessens (1982) bestätigt, daß in der Supervision der „Institutionelle Faktor" ausgeblendet wird. Aber auch dieser Befund wird indirekt erschlossen. Da sich für diesen Bereich weniger Effekte finden als in den anderen Bereichen, wird hier ein Defizit festgestellt, weil die Antworten nicht so viele positive Zustimmung fanden, wie die anderen Bereiche. Es handelt sich also wiederum um eine indirekte Vorgehensweise und nicht um eine Erhebung, die offensiv und differenziert negative Phänomene erfaßt. Möglicherweise könnte man dieses Phänomen als Verdrängung beschreiben, warum nicht offensiv das gefragt wird, was man lieber nicht wissen möchte. Hierzu gehört auch die „widersprüchliche“ Befundlage zu den Effekten auf der Klientenebene. Viele Supervisanden sehen in diesem Bereich wenig und manchmal keine positiven Auswirkungen (Beer und Gediga 1996, S. 39) und es steht $\mathrm{zu}$ vermuten, daß aus der Klientenperspektive noch weniger zu bemerken ist. Die $\mathrm{Zu}-$ sammenstellung der Befunde zu den Effekten von Supervision zur Verbesserung von 
Psychotherapie, die Luborsky et al (1988) geben, die keine Effekte nachweisen konnten, ist typisch für diesen Bereich. Möglicherweise ist dieses Ergebnis das Resultat einer zu undifferenzierten Erhebungsmethodik.

Burton, Henderson und Curtis Jenkins (1998) kommen ebenso wie Beer und Gediga (1998) zu der eindeutigen Schlußfolgerung, daß Supervision ausgesprochen hoch geschätzt wird, wenn man die direkten Kunden, die Supervisanden befragt, die auch klare positive Effekte benennen. Daneben werden eindeutig auch negative Effekte beschrieben. Mit Sicherheit kann man davon ausgehen, daß bei Supervision ein ähnliches „Varianzerweiterungsphänomen wie in der Psychotherapie auftritt, da hier ähnliche Prozesse stattfinden und gerade die interpersonellen Aspekte (Prozesse und Persönlichkeitsmerkmale) für positive Effekte verantwortlich gemacht werden.

Bergin (1963, 1967, 1971) hat sich wahrscheinlich als erster intensiv „empirisch“ mit negativen Effekten psychotherapeutischer Behandlungen beschäftigt und 30 Studien der Effizienzforschung herangezogen und an den Daten ein Phänomen beschrieben, daß als „Varianzerweiterungsphänomen“ bekannt geworden ist (z.B. Schulz 1984, S. 149). Es besagt, daß sich in den Behandlungsgruppen im Unterschied zu den Kontrollgruppen der Therapiestudien die Streuungswerte der Behandelten stärker vergrößerten, es also auch bei verbesserten Mittelwerten zu einzelnen Fällen gekommen ist, in denen sich die Werte verschlechtert haben. Bergin schätzt deshalb circa $10 \%$ der Fälle als vorwiegend verschlechtert ein (kritische Anmerkungen zur Methode Bergins in König-Fuchs 1991, S. 8). Zieht man die wenigen Befunde zu negativen Auswirkungen von Supervision heran, so kann man hier eine vergleichbare Größenordnung vermuten.

Eine Untersuchung zu den Kausalattributionen von Therapeuten, Klienten und Supervisoren zu den Gründen und Erklärungen für Mißerfolge von König-Fuchs verweist auf die Notwendigkeit sich dieser Thematik offensiv zuzuwenden, da eine Tendenz bei Therapeuten und Supervisoren besteht, Mißerfolge externalen Ursachen zuzuschreiben. Deshalb kann es nicht genügen, negative Effekte durch offene Fragen zu erfassen, die zuviel Raum für eine Verdrängung unangenehmer Wahrnehmungen lassen.

Die Erfassung unerwünschter Ereignisse, Nebenwirkungen und Wechselwirkungen hat im Bereich der Arzneimittelprüfung eine lange Tradition. Hier wurden sehr differenzierte Erfassungsstrategien entwickelt. Das „Gesetz über den Verkehr mit Arzneimitteln“ (AMG, 5. Änderungsgesetz vom 9. August 1994) und die „Allgemeine Verwaltungsvorschrift zur Anwendung der Arzneimittelprüfrichtlinien“ (in der Neufassung vom 5.5.1995) regelt das Vorgehen und legt die Standards fest. Die Vorgehensweise ist mit den EG-Regelungen harmonisiert, die im Konfliktfall übergeordnete Bedeutung haben (Kohnen et al. 1996, S. 187).

In ersten Beobachtungen wird von unerwünschten Ereignissen gesprochen, so lange noch nicht klar ist, ob sie in einem direkten Zusammenhang mit der Behandlung stehen. Der Begriff Nebenwirkung ,,ist begrenzt auf unerwünschte Begleiterscheinungen, die beim bestimmungsgemäßen Gebrauch nach der Zulassung eines Arzneimittels beobachtet werden“ (Kohnen et al 1996, S. 188). Sie werden nach Schweregraden klassifiziert und sollen ein statistisch vertretbares Auftretensrisiko nicht überschreiten. Die Anbieter eines Heilmittels sind verpflichtet, unerwünschte Ereignisse zu dokumentieren und mitzuteilen.

Von dieser Praxis sind die meisten psychosozialen Interventionsformen weit entfernt. Die Supervision bildet dabei keine Ausnahme. Trotz aller Vorbehalte gegen 
Analogien mit pharmakologischen und medizinischen Behandlungen sollten differentielle Evaluationen durchgeführt werden, die verschiedene Supervisionsmethoden hinsichtlich möglicher Nebenwirkungen vergleichen. Was sind settingspezifische Risiken und welche spezifischen Vorgehensweisen zeichnen sich durch welche Risiken für die unterschiedlichen Kunden (Klienten, Mitarbeiter, Institution etc.) von Supervision aus. Vermutlich unterschieden sich unterschiedliche Vorgehensweisen stärker durch ihre Nebenwirkungen und Risiken als durch gravierende Unterschiede im Ausmaß der Klientenzufriedenheit und der positiven Effekte.

Petzold und Rodriguez-Petzold (1997) ermittelten in einer Erhebung bei 30 Ausbildungskandidaten für Supervision und 70 der Psychotherapie, daß nur 2 aus der ersten und 4 aus der zweiten Gruppe ihre Klienten darüber informieren, daß sie ihren Fall als Lehr- oder Kontrollfall in Ausbildungssupervision einbringen (1997, S. 299f). Die Auswirkungen und die Unterschiede der Handhabung von Schweige- und Informationspflichten müssen dringend im Zusammenhang mit unerwünschten Ereignissen untersucht werden, um hier eine wahrscheinlich viel zu blauäugige Praxis weiterzuentwickeln, die den unterschiedlichen Bedürfnissen der beteiligten Personen gerecht wird. Dabei müssen methodische, ethische, klinische und juristische Perspektiven integriert werden. Keine der vier Perspektiven kann den Anspruch erheben als primäre Entscheidungsgrundlage zu dienen. Klinische und behandlungstechnische Erfordernisse können sich zum Beispiel nicht wie selbstverständlich innerhalb eines juristisch determinierten Problemfeldes bewegen, sondern müssen diese Rahmenbedingungen entsprechend des Anwendungsbereiches modifizieren (s.a. Wilker \& Höfling 1995).

Neben der Erarbeitung von Hinweisen zum Verbraucherschutz der Klienten wartet die Frage nach Kriterien für Supervisoren, wie sie Schumacher (1997) aufwirft, der für eine Checkliste plädiert, noch auf empirisch abgesicherte Antworten, damit sich nicht die Überlebensstrategien von Jay Haley (1995) bei Supervisoren durchsetzten, der einen Artikel mit der Überschrift versieht: „Über die Kunst, Supervisor zu sein, ohne zu wissen, wie man jemanden verändert“. Spezifische Leiter- und Lehrerqualifikationen (Pühl 1994) und grundsätzliche Merkmale (z.B. Albott 1984) müssen ermittelt werden, die für die vier Anwendungsbereich von Supervision eine Rolle spielen, wobei noch zu prïfen bleibt, in welchem Ausmaß die gleichen Merkmale in diesen verschiedenen Anforderungskontexten eine Rolle spielen.

\section{Brauchen wir eine Supervisionswissenschaft mit eigenen Forschungsmethoden?}

Die Supervisionsforschung steht genau wie die Sozialarbeit vor der Entscheidung, ob sie eine eigenständige Forschungsmethodologie oder sogar eine eigene Wissenschaft braucht. Angesichts der Schwierigkeiten, ihre Praxis von anderen Beratungsformen und der Psychotherapie abzugrenzen, erscheint dieser Weg wenig erfolgversprechend. Durch diese Bemühungen würde viel Energie in eine methodologische Nabelschau fließen, die wahrscheinlich besser in konkrete Praxisforschung einmünden sollte, die sich den vielen Anforderungen und Fragen der Praxis stellt, zu denen insbesondere die Beantwortung der Frage gehört, welche Grenzen und klaren Indikationskriterien es zum Einsatz von Supervision mit ihren unterschiedlichen Settings gibt. Zur Klärung 
dieser praxisrelevanten Fragen sollte der Versuchung widerstanden werden, eine Aufwertung von Supervision zu erzielen, indem man sich auf die Entwicklung abgrenzbarer Forschungsmethodologien konzentriert.

\section{Literatur}

Albott, W. (1984): Supervisory characteristics and other sources of supervision variance. The Clinical Supervisior, 2, S. 27-41.

Ambühl, H. (1994): Internationale Studie zur Entwicklung der Psychotherapeuten und Psychotherapeutinnen. Ein Forschungsprojekt des „Collaborative Research Network“ (CRN), Psychotherapeut, 39, 5, S. 336-338.

Ambühl, H., Orlinsky, D., Cierpka, M., Buchheim, P., Meyerberg, J. \& Willutzki, U. (1995): Zur Entwicklung der theoretischen Orientierung von PsychotherapeutInnen. Psychotherapie, Psychosomatik, medizinische Psychologie, 45, S. 109-120.

Auckenthaler, A. (1995): Supervision psychotherapeutischer Praxis. Organisation - Standards - Wirklichkeit. Stuttgart: Kohlhammer.

Balint, M. (1957): Der Arzt, sein Patient und die Krankheit. Stuttgart: Klett.

Bankoff, E. A., Howard, Kenneth (1992): The social network of the Psychotherapy patient and effective psychotherapeutic process. Journal of Psychotherapy Integration 2 (4), S. 273-294.

Beer, T. (1997): Evaluation von Supervision. Ein Beitrag zur Qualitätssicherung berufsbezogener Beratung. In: DGSv aktuell, 3, S. 22-23.

Beer, T. \& Gediga, G. (1998): Evaluation von Supervision: Eine Untersuchung im Bereich der Sozialen Arbeit. In: Holling, H. \& Gediga, G. (Hrsg.) Evaluation in den Arbeitswissenschaften. Göttingen (i.V., zit. nach Manuskript)

Beer, T. (1998): Evaluation von Supervision. Ein Beitrag zur Wirkungsforschung und Qualitätssicherung berufsbezogener Beratung. In: Berker, Peter \& Buer, Ferdinand (Hrsg.) Praxisnahe Supervisionsforschung. Felder - Designs - Ergebnisse. Münster: Votum, S. 99-129.

Bergin, A. E. (1963): The effects of psychotherapy: Negative results revisited. Journal of Counseling Psychology 10, S. 244-250.

Bergin, A. E. (1967): Further comments on psychotherapy research and psychotherapeutic practise. International Journal of Abnormal Psychiatry 3, S. 317-323.

Bergin, A. E (1971): The evaluation of therapeutic outcome. In: Bergin, A. E. \& Garfield, S. L. (Eds.) Handbook of psychotherapy and behavior change. New York: Wiley \& Sons, S. 217-270.

Berker, P. (1989): Das Unfertige und das Unverstandene - Versuch über den Erfolg der Supervision. Supervision, 16, S. 69-71.

Berker, P. \& Buer, F. (Hrsg.)(1998): Praxisnahe Supervisionsforschung. Felder - Designs - Ergebnisse. Münster: Votum.

Bernard, J. M. \& Goodyear, R. K. (1992): Fundamentals of clinical supervision. Boston: Allyn \& Bacon.

Blocher, D. H. (1983): Toward a Cognitive Developmental Approach to Counseling Supervision. The Counseling Psychologist, 11, S. 27-34.

Brodsky, A. M. (1980): Sex role issues in the supervision of therapy. In: Hess, A. K. (Ed.): Psychotherapy supervision: Theory, research and practice. New York: Wiley, S. 509-522.

Buer, F. (1998) Einführung. In: Berker, P. \& Buer, F. (Hrsg.) Praxisnahe Supervisionsforschung. Felder Designs - Ergebnisse. Münster: Votum, S. 8-13

Bundesinstitut für Arzneimittel und Medizinprodukte (1995): 3. Bekanntmachung über die Anzeige von Nebenwirkungen, Wechselwirkungen mit anderen Mitteln und Arzneimittelmißbrauch nach $\$ 29$ Abs. I Satz 2 bis 8 AMG (Entwurf vom 24.1.1995).

Bundesministeriums für Familie, Senioren, Frauen u. Jugend (Hrsg.)(1994a): Beratungsbegleitende Forschung und Evaluation von Vorgehensweisen in der Ehe-, Familien- und Lebensberatung (Herausgeber des Bandes: Klann, Notker \& Hahlweg, Kurt) Stuttgart: Kohlhammer.

Bundesministeriums für Familie, Senioren, Frauen u. Jugend (Hrsg.)(1994b): Bestandsaufnahme in der Institutionellen Ehe-, Familien- und Lebensberatung (Herausgeber des Bandes: Klann, Notker \& Hahlweg, Kurt) Stuttgart: Kohlhammer.

Burton, M., Henderson, P. \& Curtis Jenkins, G. (1998) Primary Care Counsellors` Experiences of Supervision. Counselling May 1998, S. 122-133. 
Cloetta, B. (1985): Praxisberatung von Lehrer/innen: Erst qualitative empirische Ergebnisse. In: A. Schorr (Hg.): Bericht über den 13. Kongreß für Angewandte Psychologie. Bonn: Deutscher Psychologen Verlag.

Conen, M. L. (1993): Frauen und Männer in Supervision - Welchen Unterschied macht das? In: H. Neumann-Wirsig \& H. Kersting (Hg.): Systemische Supervision oder: Till Eulenspiegels Narreteinen. Schriften zur Supervision, 4, S. 205-224.

Crits-Christoph, P. \& Mintz, J. (1991): Implications of therapist effects for the design and analysis of comparative studies of psychotherapies. Journal of Consulting and Clinical Psychology 59 (1), S. 20-26.

Crits-Christoph, P., Baranackie, J. S. K., Beck, A. T., Carroll, K,, Perry, K., Luborsky, L., McLellan, A. Th., Woody, G. E., Thompson, L., Galagher, D. \& Zitrin, Ch. (1991): Meta-analysis of therapist effects in psychotherapy outcome studies. Psychotherapy Research 1 (2), S. 81-91

Denner, L. (1998): Supervision und Pädagogische Fallbesprechungen an Schulen - Entwicklung und Design einer Evaluationsstudie. In: P. Berker \& F. Buer (Hg.). Praxisnahe Supervisionsforschung. Münster: Votum, S. 148-182.

De Lorent, H. P. (1992): Praxisschock und Supervision. Auswertung einer Umfrage bei neu eingestellten Lehren. Pädagogik, 44, S. 22-25.

EG-Kommision Direktorat III (1994): Notice to applicants for marketing authorisations for medical products for human use in the European Community, December 1994 (III/5944/94).

Elbing, E. \& Huber, U. (1988): Supervision als Programmatik und als subjektiv erfahrene Alltagsrealität. Eine qualitative Pilotstudie bei Mitarbeitern in Erziehungsberatungsstellen. Psychologische Arbeiten und Berichte (PAB), 23.

Erger, R. \& Molling, M. (1991): Der kleine Unterschied - Frauen und Männer in der Supervision. Hille: Ursel Busch Fachverlag.

Felbermeyer, St. (1996): Evaluation von Team-Supervision bei Krankenhauspflegepersonal. Unveröff. Diplomarbeit, TU Berlin Klinische Psychologie.

Fengler, J. (1994): Helfen macht müde. Zur Analyse und Bewältigung von Burnout und beruflicher Deformation. München: Pfeiffer 3. erw. Auflage.

Filsinger, D. \& Schäfer, J. (1992): Der institutionelle Handlungskontext als Gegenstand von Supervision und Organisationsberatung. In: A. Auckenthaler \& D. Kleiber (Hg.). Supervision in Handlungsfeldern der psychosozialen Versorgung. Tübingen: DGVT-Verlag, S. 78-100.

Filsinger, D. (1992): Der institutionelle Handlungskontext als Gegenstand von Supervision und Organisationsberatung. In: A. Auckenthaler \& D. Kleiber ( $\mathrm{Hg}$.). Supervision in Handlungsfeldern der psychosozialen Versorgung. Tübingen: DGVT-Verlag, S. 78-100.

Friedlander, M. L. \& Ward, L. G. (1984): Development and validation of Supervisory Styles Inventory. Journal of Counseling Psychology, 31, S. 541-557.

Goodyear, R. K.; Abadie, P. D. \& Efros, F. (1984): Supervisory theory into practice: Differential perception of supervision by Ekstein, Ellis, Polster and Roger. Journal of Counseling Psychology, 31, S. 228237.

Grawe, K. (1998): Psychologische Therapie. Göttingen: Hogrefe.

Greffrath, M. (1998): „Risiko heißt: Eine Entscheidung kann noch fallen!“ Körber Stiftung (Hrsg.) Deutscher Studienpreis Risiko! Der Umgang mit Sicherheit, Chance und Wagnis. Hamburg: Körber Stiftung, S. 10-13.

Haley, J. (1995): Über die Kunst, Supervisor zu sein, ohne zu wissen, wie man jemanden verändert. Z.system.Ther. 13 (1), S. 39- 50 (orig. Journal of Systemic Therapy (JST) Winter 1993, S. 41-52).

Hege, M. (1990): Männer und Frauen in der Supervision. Supervision Sonderheft, S. 33-43.

Hempel, C. G. (1966): Philosophy of natural science. Englewood Cliffs, N.J.: Prentice-Hall. (Philosophie der Naturwissenschaften. München: DTV 1974).

Henry, W. P., Strupp, H. H., Butler, S. F., Schacht, Th. E., Binder, J. (1993): Effects of Training in TimeLimited Dynamic Psychotherapy: Changes in Therapist Behavior. Journal of Consulting and Clinical Psychology 61 (3), S. 434-440.

Heppner, P. P. \& Handley, P. G. (1982): The relationship between supervosory behavior and perceived supervisor expertness, attractiveness and trustworthiness. Counselor Education and Supervision, 22, S. 37-45.

Heppner, P. P. \& Roehlke, H. J. (1984): Differences Among Supervisees at Different Levels of Training: Implications for a Developmental Model of Supervision. Journal of Counseling Psychology, 31, S. 7690.

Hogan, R. A. (1964): Issues and Approaches in Supervision. Psychotherapy: Theory, Research and Practice, 1, S. 1739-1741. 
Holloway, E. L.; Freund, R. D.; Gardner, S. L.; Nelson, M. L.\& Walker, B. R. (1989): Reelations of Power and involvement to theoretical orientation in supervision: An analysis of discourse. Journal of Counseling Psychology, 36, S. 88-102.

Howard, K.I., Lueger, R.J., Maling, M.S. \& Martinovich, Z. (1992): A phase theory of psychotherapy. Annual International Meeting of the Society for Psychotherapy Research, Berkeley, USA.

Howard, K. I., Kopta, S. M., Krause, Merton S. \& Orlinsky, D. E. (1986): The dose-effect relationship in psychotherapy. American Psychologist 41 (2) S. 159-164.

Hunt, D. E. (1971): Matching Models in Education: The Coordinatopn of Teaching Methods with Student Characteristics. Toronto: Ontario Institute of Studies in Education.

Jacobson, N. S. (1997): Psychotherapy research in transition: Implications for Psychotherapy integration. Society for the Exploration of Psychotherapy Integration: Thirteenth Annual Conference „Embracing New Approaches", Toronto, Canada, 24.-27.4.

Jacobson, N. S. \& Truax, P. (1991): Clinical significance: A statistical approach to defining meaningful change in psychotherapy research. Journal of Consulting and Clinical Psychology 59 (1), S. 12-19.

Kessel, L.van (1998): Theorie und Praxeologie des Lernens in der Supervision. Einige Bausteine aus niederländischer Sicht. In: Berker, P. \& Buer, F. (Hrsg.) Praxisnahe Supervisionsforschung. Felder - Designs - Ergebnisse. Münster: Votum, S. 46-68.

Kinzl, J. (1990): Langjährige Balint-Gruppe mit Logopädinnen. Erfahrungen und Veränderungen. Sprache - Stimme - Gehör, 14, S. 120-123.

Kohnen, R., Fichte, K., Hofmann, S., Kastrup, H. \& Meng, G. (1996): Erfassung unerwünschter Ereignisse und ihre Bewertung als unerwünschte Arzneimittelwirkungen in der Entwicklung von Therapien. In: Collegium Internationale Psychiatriae Scalarum (Hrsg.) Internationale Skalen für Psychiatrie, 4. überarb. Auflage. Weinheim: Beltz, S. 187-201.

König-Fuchs, C. (1991): Therapeutischer Erfolg und Mißerfolg. Kausalattributionen von Therapeuten, Klienten und Supervisoren. Frankfurt/M: Peter Lang

Kühl, W. \& Pastäniger-Behnken, C. (1998): Supervision in Thüringen - Design einer Erkundungs- und Evaluationsstudie im Bereich der Sozialen Arbeit. In: P. Berker \& F. Buer (Hg.). Praxisnahe Supervisionsforschung. Münster: Votum, S. 130-147.

Lambert, M. J., Bergin, A. E. (1994): The effectivieness of psychotherapy. In: Garfield, Sol. L. \& Bergin, Allen E. (Eds) Handbook of psychotherapy \& behavior change 4 ed. New York: John Wiley \& Sons, S. 143-189.

Luborsky, L., Crits-Christoph, P., Mintz, J. \& Auerbach, A. (1988): Wo will benefit from Psychotherapy? Predicting therapeutic outcomes. New York: Basic Books.

Marikis, D. A., Russell, R. K. \& Dell, D. M. (1985): Effects of supervision experience level of planning and insession supervisor verbal behavior. Journal of Counseling Psychology, 32, S. 410-416.

Möller, H., Märtens, M.(1998) Supervisionsforschung ohne Zukunft? Supervision als homöopathische Inszenierung, OSC 3

McNeill, B.; Stoltenberg, C. D. \& Pierce, R. A. (1985): Supervisees Perception of their Developement: A Test of the Counselor Complexity Model. Journal of Counseling Psychology, 32, S. 630-633.

Meidinger, H. (1991): „Schulhausinterne Supervision“ - ein Beispiel systembezogener Beratung in der Schule. Psychologie in Erziehung und Unterricht, 38, S. 292-297.

Miars, R. D.; Tracey, T. J.; Ray, P. B.; Cornfeld, J. L.; OíFarell, M. \& Gelso, C. J. (1983): Variations in Supervision Process Across Trainee Experience Levels. Journal of Counseling Psychology, 30, S. 403-412.

Mutzeck, W. (1988): Von der Absicht zum Handeln. Rekonstruktion und Analyse Subjektiver Theorien zum Transfer von Fortbildungsinhalten in den Berufsalltag. Weinheim: Deutscher Studien Verlag.

Nellessen, L. (1982): Supervision - Vom Drauf-schauen übers Vor-machen zum Mittun! In: Gesamthochschule Kassel (Hrsg.) Beiträge zur Supervision. Band 1, Kassel: Gesamthochschule Kassel, S. 46-66.

Nelson, M. L. \& Holloway, E. L. (1981): Relation of gender to power and involvement in supervision. Journal of Counseling Psychology, 37, S. 473-481.

Orlinsky, D. E. \& Howard, K. I. (1986): Process and outcome in psychotherapy. In: Garfield, Sol. L. \& Bergin, Allen E. (Eds) Handbook of psychotherapy \& behavior change 3 ed. New York: John Wiley \& Sons, S. 311-384.

Petty, M. \& Odewahn, C. (1984): Supervising behavior and sex role stereotypes in human services organizations. The Cinical Supervisor, 1, S. 13-20.

Petzold, H. \& Schigl, B. (1996): Evaluation einer Supervisorenausbildung für den sozialgerontologischen und Krankenhausbereich. Forschungsbericht. Wien: Karl Kummer Institut. 
Petzold, H. G. \& Rodriguez-Petzold, F. (1997): Anonymisierung und Schweigepflicht in supervisorischen Prozessen - ein methodisches, ethisches, klinisches und juristisches Problem. Familiendynamik 22 (3), S. 288-311.

Petzold, H., Hass, W. Jakob, S., Märtens, M. \& Merten, P. (1998): Qualitätssicherung durch Ausbildungsevaluation in der Integrativen Therapie. In: Laireiter, Anton \& Vogel, Heiner (Hrsg.) Qualitätssicherung in der Psychotherapie - Ein Handbuch. Tübingen: DGVT-Verlag

Pühl, H. (Hrsg.) (1994): Handbuch der Supervision 2. Berlin: Edition Marhold im Wissenschaftsverlag Volker Spiess.

Pühl, H. (1994 a): Der Supervisor als Lehrer und Leiter. In: Pühl, H. (Hrsg.) Handbuch der Supervision 2. Berlin: Edition Marhold im Wissenschaftsverlag Volker Spiess, S. 353-364.

Putney, M. W.; Worthington, E. L. \& McCullough, M. E. (1992): Effects of supervisor and supervisee theoretical orientation and supervisor-supervisee matching on interns' perception of supervision. Journal of Counseling Psychology, 39, S. 258-265.

Reising, G. N. \& Daniels, M. H. (1983): A study of Hogan`s model of counselor develpment and supervision. Journal of Counseling Psychology, 30, S. 235-244.

Retzer, A., Schumacher, B., Weber, G. \& Fischer, H.-R. (1997): Zur Form systemischer Supervision. Familiendynamik 22 (3), S. 240-263.

Roth, A. \& Fonagy, P. (1996): What works for whom? A critical review of psychology research. New York: Guilford Press.

Schneider, K. \& Müller, A. (1995): Evaluation von Supervision. Supervision, 27, S. 86-98.

Schreyögg, A. (1994) Supervision: Didaktik \& Evaluation. Integrative Supervision in der Praxis. Paderborn: Junfermann Verlag

Schulz, W. (1984): Analyse negativer Therapieeffekte und Probleme der Kontraindikation. In: Jüttemann, G. (Hrsg.) Neue Aspekte klinisch-psychologischer Diagnostik. Göttingen: HogrefeVerlag für Psychologie D.C.J..

Schumacher, B. (1997): Ist Ihr Supervisor verkehrssicher? - Plädoyer und Checkliste für einen Supervisions-TÜV. Familiendynamik 22 (3), S. 312-315.

Shapiro, D. A., Harper, H., Startup, M., Reynolds, S., Bird, D. \& Suokas, A. (1994): The high-water mark of the drug metaphor: A meta-analytic critique of process-outcome research. In: Russell, Robert L. (Ed) Reassessing psychotherapy research. New York: Guilford, S. 1-35.

Stein, D.M. \& Lambert, M..J. (1995): Graduate training in psychotherapy: Are therapy outcomes enhanced? Journal of Consulting and Clinical Psychology 63 (2), S. 182-196.

Steinhelber, J.; Patterson, V.; Cliffe, K \& LeGoullon, M. (1984): An investigation of some relationsships between psychotherapy supervision and patient change. Journal of Clinical Psychology, 40, S. 13461353.

Stoltenberg, C. D. (1981): Approaching Supervision from a Developmental Perspektive: The Counselor Complexity Model. Journal of Counseling Psychology, 28, S. 59-65.

Strauß, B. (1998) Evaluation in der stationären Psychotherapie. Expertentagung: Wirksamkeitskriterien in der Psychotherapie. Köln am 7.5.98

Volkart, R. (1995): Patient und Therapeut zwischen Skylla und Charybdis! Die Interpretation eines Transskriptes mit dem Psychotherapie-Prozeß-Modell von Joseph Weiss. In: M. B. Buchholz. Psychotherapeutische Interaktion. Opladen: Westdeutscher Verlag: S. 127-173.

Wampold, B. E. (1997): Methodological problems in identifying efficacious psychotherapies. Psychotherapy Research 7, (1) S. 21-44.

Ward, L. G.; Friedlander, M. L.; Schoen, L. G. \& Klein, J. G. (1985): Strategic self-presentation in supervision. Journal of Counseling Psychology, 32, S. 11-118.

Weiss, J. (1993): How psychotherapy works. Prozess and technique. New York: Guilford.

Wilker, F.-W. \& Höfling, S. (1995): Praxisorientierte ethische und juristische Aspekte supervisorischen Handelns. In: Wilker, F.-W. (Hrsg.)(1995) Supervision und Coaching. Aus der Praxis für die Praxis. Bonn: Deutscher Psychologen Verlag, S. 275-282.

Willutzki, U. (1995): NovizInnen und erfahrene TherapeutInnen: Brauchen alle dieselbe Supervisionsform? Verhaltenstherapie und psychosoziale Praxis, 3, S. 419-435.

Worthington, E. L.\& Stern, A. (1985): Effects of supervision and supervisee degree level and gender on the supervisory relationship. Journal of Counseling Psychology, 32, S. 252-262.

Zielke, M. (1982): Supervision. In: Bastine R., Fiedler; P., Grawe, K., Schmidtchen, S. \& Sommer, G. (Hrsg.) Grundbegriffe der Psychotherapie. Weinheim: Edition Psychologie, S. 403-406. 